\title{
Congenital Missing Teeth
}

\author{
Jessica Anggono ${ }^{1}$, Elza Ibrahim Auerkari ${ }^{2 *}$ \\ ${ }^{1}$ Pediatric Residency Program, Faculty of Dentistry, University of Indonesia, Jakarta 10430, \\ Indonesia \\ ${ }^{2}$ Department of Oral Biology, Faculty of Dentistry, University of Indonesia, Jakarta 10430, \\ Indonesia \\ *Email: elza.ibrahim@ui.ac.id
}

\begin{abstract}
Oral health plays a major role in an individual's well-being, affecting all aspects of quality of life: esthetics, function, and speech. Abnormalities in oral health may disrupt any or all of these aspects. One of the challenging and most common dental anomalies found in daily practice is congenital missing teeth (CMT). CMT affects more than $20 \%$ of the human population and can negatively affect mouth esthetics, dental function, and even a person's speech. CMT essentially result from a defect in tooth development, which is a complex process. Environmental and genetic factors, combined with epigenetics, have been credited with disrupting tooth development, causing CMT. Most cases of CMT are found in a familial pattern, and among all factors, genetics plays a fundamental part; therefore, heredity has a crucial role in determining an individual's phenotype of CMT. There are more than 300 genes that contribute to odontogenesis, and their mutations may be manifest as hypodontia with varying degrees of penetrance and expressivity. A major cause of autosomal dominant non-syndromic hypodontia is mutation in the MSX1 and PAX9 genes in humans. In the present paper, we will discuss in brief the definition, prevalence, etiology, and roles of genetics and epigenetics in CMT, as well as the types of gene mutation, with emphasis on mutation in the MSX1 and PAX9 genes related to CMT.
\end{abstract}

Keywords: congenital missing teeth, genetics, gene mutations

\section{Introduction}

One of the most common dental anomalies is congenital absence of teeth, also known as congenital missing teeth (CMT), congenital dental aplasia, dental or tooth agenesis, usually referred to as hypodontia [1]. The ideal diagnosis for CMT requires x-rays and a clinical examination, sometimes including a dental cast, but above all, the Roentgen examination is an essential part of CMT diagnosis, because radiography can reveal the presence of tooth germs [1]. All of the deciduous teeth and the first permanent molar buds are visible by Roentgen examination at birth, and the premolar crowns and second permanent molars begin the mineralization process around the age of 2 years. All of the permanent teeth except the third molars have begun their mineralization stage by the age of 6 years [2]. Therefore, without radiography, a clinical examination for CMT in the case of primary dentition can be performed at the age of 3-4 years when all of the primary teeth are already erupted, whereas for CMT in the case of permanent dentition, the diagnosis can be 
determined between the ages of 12 and 14 years, when all of the succedaneous teeth are already erupted, excluding the third molars [2].

Although no precise definition or classification of CMT exists in the literature, the following definitions have been widely used, based on the number of missing teeth: oligodontia, hypodontia, and anodontia [3]. Oligodontia is defined as the congenital absence of more than six teeth, excluding the third molars [3]. Hypodontia is described as congenital absence of 1-6 teeth, excluding the third molars [3]. Lastly, anodontia is known as the complete absence of teeth on one or both dentitions [3].

Hypodontia and oligodontia are also divided into non-syndromic (isolated) and syndromic (related to their syndromes) types [4]. This anomaly is associated with at least 49 syndromes registered in the Online Mendelian Inheritance in Man Database (OMIM) [4]. Moreover, hypodontia can occur together with other dental anomalies such as macrodontia, anomalies in morphology, taurodontism, enamel hypoplasia, short roots, ectopic eruption, microdontia, and most commonly, tapering or peg-shaped teeth $[4,5]$.

\subsection{Prevalence}

CMT is more commonly found in permanent than in primary dentition [1]. The prevalence rate of CMT in the deciduous dentition is between $0.1 \%$ and $2.4 \%$ [1]. In the permanent dentition, the prevalence of CMT without the third molars varied between $0.15 \%$ and $16.2 \%$ in studies with sample sizes ranging from 200 to greater than 100,000 subjects [1]. However, hypodontia in the deciduous dentition is usually followed by hypodontia in the succedaneous dentition [1]. The teeth most commonly affected by this anomaly, not including the third molars, are the mandibular second premolars followed by the maxillary lateral incisors and second premolars $[5,6]$. Women are usually more affected than men, with a male-to-female ratio of approximately 2:3. The prevalence of bilateral missing teeth is more common than that of unilateral missing teeth [1].

CMT affects more than $20 \%$ of the human population [7]. The prevalence of CMT varies between continents and races [1]. The highest prevalence of missing deciduous and permanent dentition is seen in Japanese people [1]. The prevalence of CMT in the European Caucasian population ranges from $4 \%$ to $8 \%$ [3]. The use of different measurement approaches or other methodologies and ethnic background diversity could cause variation in the prevalence of CMT [1]. Generally, the prevalence of CMT increases over time, possibly due to evolutionary changes, such as shrinking of the mandible, or to an increase in the diagnosis rate of CMT [1].

\subsection{Etiology}

CMT can be caused by disturbances during the early stages of development and can result in a mild dysplastic expression of the ectoderm [1]. Several theories exist about tooth agenesis: decreasing jaw size throughout human evolution, increased susceptibility to agenesis in an area or 'fragile site' due to epigenetic influences, the last field of neural developmental being the most likely linked to tooth agenesis, and insufficient primordia for tooth germ initiation [8]. Studies on monozygotic twins have confirmed that inheritance is one of the major causative factors in CMT 
[1]. The existence of different patterns of CMT between monozygotic twins might be due to underlying epigenetic factors [1]. Other factors may include external influences or a combination of environmental factors, such as infection, fractures, surgical procedures, traumas, drugs, chemotherapy, radiotherapy, and genes associated with approximately 120 syndromes, including cleft palate, cleft lip, or both; ectodermal dysplasia; and Down, Book and Rieger syndromes [1, 5]. Another study showed that a lack of space for tooth development and decreased tooth size are correlated with CMT and late tooth development, for example, disturbance of tooth agenesis associated with maternal systemic disease, diabetes, and different infections such as maternal rubella infection during pregnancy [1]. Moreover, it is stated that hypodontia in the anterior dentition may depend more on genes, whereas posterior missing teeth may be sporadically caused by environmental and genetic factors, although the exact mechanisms are unknown $[1,9]$.

\section{Role of Genes in Tooth Development}

Odontogenesis is a complex process associated with genes that specify the size, location, and type of each tooth organ, as well as the processes of amelogenesis and dentinogenesis [3]. Failure of tooth formation in the majority of cases is correlated with genetic background. Due to its genetic basis, CMT is more likely to be found in relatives of persons with CMT than in a randomly chosen population [4]. During intrauterine development, differentiation and morphogenesis are the result of complex interactions at the molecular level between the mesenchyme and the ectoderm [3]. Numerous different genes have been implicated in tooth development by experimental studies and gene expression analyses [3]. To date, more than 200 genes involved in the embryonic development, differentiation, and morphogenesis of teeth have been identified [3]. Different but related genes, such as MSX1 (4p16.1-located on chromosome no. 4 short arm band 16 and sub-band 1), PAX9 (14q12-13-located on chromosome 14 long arm band 12 to 13), ectodysplasin-A (EDA), axis inhibition protein 2 (AXIN2), bone morphogenetic proteins (BMP), fibroblast growth factors (FGFR1), TGFA, ectodysplasin-A receptor (EDAR), IRF6, Wingless (WNT10A), 10q11.2 (located on chromosome no. 10 long arm band 11 and sub-band 2), 16q12.1 (located on chromosome no. 16 long arm band 12 and sub-band 1), and others may be associated with defects in tooth development [7]. Signaling molecules that determine the shape and position of the teeth are DLX1, DLX2, BARX1, MSX1, MSX2, and PAX.10 CMT is a result of impaired gene function that manifests as impaired signal regulation or reduced signaling and defects in cell migration, proliferation, and differentiation [3].

CMT may occur in isolated cases and syndrome types, where the isolated case is the common one and may be familial or sporadic [1]. Non-syndromic hypodontia has been reported with autosomal recessive (AR), autosomal dominant (AD), polygenic, and X-linked patterns of inheritance, with wide variation in both penetrance (some individuals have no disease even though the individual carries the gene defect or genotype associated with the disease) and expressivity (different phenotypes among individuals) $[1,4]$. Carriers in the same family may exhibit significant variability in symmetry, location within the dental arch, and the number of teeth [11]. 
Of all mutations associated with the autosomal dominant, non-syndromic form of CMT in hypodontia, those in MSX1 and PAX9 are the most intensively studied $[1,3,4]$. The MSX1 and PAX9 genes are expressed in the mesenchyme of odontogenic sites, especially in developing tooth germs in the cap and bud stage, as a response to epithelial signals [5]. PAX9 and MSX1 are examples of controlling factors during the odontogenic process and the key to maintaining odontogenic potential [4]. Interaction between these two genes generates the ability of PAX9 to activate both mesenchymal BMP4 (bone morphogenetic protein 4) and MSX1 gene expression during tooth development and drives the morphogenesis of the dental organ - the transition from the bud stage to the cap stage during odontogenesis [3]. One of the critical factors in the signaling pathway is BMP4, which is involved in PAX9 and MSX1 function [9]. MSX1 interacts with PAX9 at both the protein and gene levels, and this interaction enhances the ability of PAX9 to regulate BMP4 expression and transactivate MSX1 [9]. Besides BMP4 downregulation, selective reduction in PAX9 binding to sites that regulate MSX1 expression levels could be a result of mutation in PAX9 [10]. Haploinsufficiency of these genes may cause more severe forms of hypodontia than point mutations in the gene [4]. Moreover, studies show that alteration of MSX1 is generally associated with the autosomal dominant inheritance of hypodontia, while defects in PAX9 are associated with hypodontia and reduction in tooth size [7].

\subsection{Gene Mutation}

A permanent alteration in the DNA sequence of a gene, such that the sequence differs from that of the most common form is referred to as a gene mutation [12]. There are two major classifications of gene mutations, hereditary mutations and acquired or somatic mutations [12]. Hereditary mutations are present throughout a person's life in every cell in the body and inherited from a parent [12]. In contrast, somatic or acquired mutations occur at some time during a person's life and are present only in certain cells, due to a mistake in DNA replication during cell division or due to environmental factors [12]. The next generation will not inherit acquired mutations [12]. Mutations in the form of nucleotide changes in genes result in malfunctioning proteins with altered protein interactions and/or structure [9]. The organs and cells requiring the function of these proteins may fail to function properly, leading to one or more missing teeth [9].

\subsection{Types of Mutation}

The DNA sequence of a gene can be altered in many ways [12]. Various gene mutations have effects on health, depending on whether they alter the function of essential proteins and where they occur [12]. Based on the nature of the nucleotide change, there are two types of mutation, transitions and transversions [13]. Substitution of a purine for another purine nucleotide (adenine to guanine) or a pyrimidine for another pyrimidine nucleotide (cytosine to thymine) is called a transition mutation [13]. The substitution of a purine for a pyrimidine or a pyrimidine for a purine is referred to a transversion mutation [13]. Transversion and transition mutations are both classified as point or missense mutations when a single nucleotide change likely causes substitution by a different amino acid [13]. A point mutation changes 
the sequence of a codon in the message by substituting one letter with another [14]. A point mutation can be of the missense, nonsense, or silent type. The three types of point mutation are defined as follows 12:

- A change in one DNA base pair that results in the substitution of one amino acid for another in the protein made by a gene is called a missense mutation.

- A change in one DNA base pair resulting in a stop codon is called a nonsense mutation. Rather than substituting one amino acid for another, the altered DNA sequence prematurely signals the cell to stop building a protein. This type of mutation may result in a shortened protein that may function improperly or not at all.

- A single nucleotide substitution that does not change the sequence of the protein is called a silent mutation.

- In addition to point mutations, there are three types of mutation involving either addition or deletion of nucleotide bases:

- Changes in the number of DNA bases in a gene by addition of a piece of DNA are called insertion mutations. The protein made by the gene may not function properly.

- A change in the number of DNA bases by removal of a piece of DNA is called a deletion mutation. Small deletions may remove one or a few base pairs within a gene, while removal of an entire gene or several neighboring genes may be seen in larger deletions. The function of the resulting protein may be altered by the deleted DNA.

- A piece of DNA that is abnormally copied one or more times called a duplication mutation. The function of the resulting protein may be altered from this type of mutation.

When the addition or loss of DNA bases shifts the grouping of bases, thus changing a gene's reading frame, a frameshift mutation occurs. The resulting protein is usually nonfunctional. Deletions, insertions, and duplications may or may not be frameshift mutations.

A short DNA sequence that is repeated several times in a row is called a repeat expansion mutation. The number of repeats can be varied, for example, a tetranucleotide repeat is made up of 4-base-pair sequences, and a trinucleotide repeat is made up of 3-base-pair sequences. A repeat expansion is a mutation that increases the number of times that the short DNA sequence is repeated. The resulting protein may function improperly due to this type of mutation.

\section{Mutation in the MSXI Gene}

One of the homeobox genes is MSX1 (muscle segment homeobox 1), which is located on chromosome 4 (4p16.1). MSX1 encodes a 60-amino-acid DNA-binding protein.3,15 The two exons are the protein-encoding portion of the gene, 1214 and $590 \mathrm{bp}$ in length, and separated by a 2107-bp intron, the second exon of the homeodomain [13]. A protein structural domain that binds RNA or DNA and is thus found in transcription factors in general is called a homeodomain [13]. The main function of the MSX1 protein is to increase the rate of transcription by interacting with transcription factors [7]. MSX1 is highly expressed in the dental mesenchyme 
and regulates tooth shape and position $[5,10]$. The MSX1 protein is considered have a crucial role during early tooth development; it was found to regulate other genes involved in tooth development pathways and to possess sequence specific DNAbinding activity [3]. Five-point mutations have been identified associated with tooth agenesis within MSX1 gene $[4,10]$. Two of these mutations are in the N-terminal region (beginning end of the protein) prior to the homeodomain (S105K and M61K), and the other three mutations (R196P, Q187X, and S202X) are located within the homeodomain area [4].

The M61K mutation (methionine to lysine at amino acid position 61) is one of the two substitution mutations located outside the homeodomain of the MSX1 gene [4]. Patients with oligodontia show a transversion mutation from thymine to adenine at codon 182, resulting the amino acid substitution M61K [7]. The effect of this mutation is still unknown, but evidence suggests that it is related to disruption of protein interactions [4]. The other substitution mutation that can be found in the MSX1 gene is the missense mutation replacing guanine with cytosine at codon no. 587 in the homeodomain area, resulting in the amino acid substitution R196P (arginine to proline at position 31 ). This mutation can disturb the stability and functional activity of the protein and is associated with a severe form of autosomal dominant oligodontia [4, 7]. Oligodontia was transmitted to four generations of a family in an autosomal dominant inheritance pattern [3]. This missense mutation was found in a family with symmetric, bilateral missing teeth involving the third molars and second premolars; some also presented phenotypic variability in the form of missing mandibular first permanent molars or maxillary first premolars [16]. In contrast, a study found that the mutations M61K and R196P were responsible for a specific pattern of severe tooth agenesis involving the third molars and second premolars but not the maxillary molars [16].

On the other hand, S105X is the only one of the three premature termination mutations that is found before the MSX1 homeodomain area [4]. In a study, a Dutch family with an autosomal dominant trait suffering from cleft lip-palate and hypodontia had the S105X (serine to an unknown amino acid) mutation caused by the $314 \mathrm{C}>$ A nucleotide substitution from cytosine to adenine at nucleotide 314 introducing a stop codon within exon 1 prior to the MSX1 homeodomain $[4,7,16]$. The premature termination caused a nonsense mutation, which led to severe tooth abnormalities, such as non-syndromic cleft palate and cleft lip [7].

Two other termination mutations fall within the homeodomain.4 One is caused by a $559 \mathrm{C}>\mathrm{T}$ nucleotide substitution of cytosine to thymine at nucleotide 559 , where it forms a stop codon that terminates the protein in the MSX1 homeodomain. This mutation was found in a Flemish family suffering from cleft lip-palate and hypodontia [4]. Lastly, in a patient with Witkop syndrome who also had hypodontia, the S202X mutation, which is caused by the nucleotide substitution $605 \mathrm{C}>\mathrm{A}$ (cytosine to adenine at nucleotide 605), was found [4]. This mutation resulted in a lack of the required protein (haploinsufficiency). Whereas a stop codon in the homeodomain region results in a premature termination and a truncated protein, substitution mutations change MSX1 activity without abolishing it completely; therefore, truncation mutations lead to a more severe phenotype in which MSX1 activity is abolished $[4,7]$. A study showed that autosomal dominant tooth agenesis, orofacial 
clefting, and the Witkop syndrome nonsense mutation were capable of producing a variable degree of maxillary molar agenesis [16]. However, the relation between the severity of hypodontia and the severity of the effect on the MSX1 protein caused by the identified missense mutations is still unclear [4].

Numerous cases of hypodontia were not related to MSX1 defects [16]. Different types of agenesis (incisor, premolar, or canine) unrelated to MSX1 defects were found in five families, demonstrating a contribution to the clinical variation in hypodontia associated with different genes [6]. The pattern of dental agenesis within a family is non-uniform: it may vary in morphology and number, i.e., premolar agenesis, incisor agenesis, and conically shaped teeth [16]. Gene carriers might exhibit reduce penetrance and no hypodontia phenotype in some families [16].

\subsection{Mutation in the PAX9 Gene}

PAX9 (paired box 9), a member of the paired box gene family, encodes transcription factors involved in positioning and morphogenesis of the entire dentition and has a major role in embryogenesis [7]. It is expressed in the specific prospective sites of all teeth prior the signs of embryogenesis; therefore, it establishes the location and the moment to begin the odontogenesis process $[5,10]$. PAX9 is located in 14q12-q13 (chromosome 14 long arm number 12 to 13) [15]. PAX genes encode 128-amino-acid proteins, including those found in eukaryotic transcription regulatory proteins involved in embryogenesis [3]. Defects in the PAX9 gene are mainly related to sporadic agenesis of the second premolars, lower permanent lateral incisors, and permanent molars [16]. Frameshift and missense mutations have been related to PAX9 in association with dental agenesis [4]. Of seven missense mutations in the PAX9 gene, six are substitution mutations, including five substitutions in the protein (K91P, R28P, R26W, G51S, and L21P), one that prevents PAX9 expression $(1 \mathrm{~A}>\mathrm{G})$, and one premature termination (K114X) [4]. Three frameshift mutations have been found in PAX9 associated with hypodontia: one of them is the deletion of eight nucleotides with the insertion of 288 foreign nucleotides (R59fsX177) at the $\mathrm{C}$-terminal region of the PAX9 paired domain, and two of them are caused by the insertion of a single nucleotide (G73fsX316 and V265fsX316) [4]. A study by Mostowska et al. showed that a frameshift mutation detected in a family with hypodontia affects hair development and missing teeth in humans and mice [9].

All of the mutations occur within the $\mathrm{N}$-terminal paired domain except V265fsX316, which is near the middle of the C-terminal region [4]. The insertion of a single nucleotide located in exon 4 of the PAX9 gene causes a premature truncation of the protein due to a frameshift at amino acid 264 [4]. Elsewhere, the insertion of 5-6 guanine nucleotides at G73fsX316 causes a severe frameshift mutation between the $\mathrm{C}$ - and $\mathrm{N}$ - terminal DNA binding domains of the PAX9 paired domain [4]. A 288-bp insertion (R59fsX177) in place of eight deleted nucleotides in the paired domain of PAX9 is the most severe of all frameshift mutations [4]. Two nucleotide substitutions (K91E and L21P) were found in another study, both of which were located in the PAX9 paired domain and affected transcriptional activity [4].

The nucleotide switch A340T (adenine transverse to thymine at nucleotide 340) at lysine 114 in the end of the N-terminal region of the PAX9 paired-box domain A 
causes a substitution mutation at K114X (lysine to an unknown amino acid) resulting in a premature termination that generates a shorter PAX9 protein [4, 7]. Lastly, three other missense mutations that lead to a residue substitution in the PAX9 protein (R26W, R28P, and G51S) were discovered [4]. An R26W (transition cytosine to thymine at $\mathrm{C} 76 \mathrm{~T}$ and the replacement of arginine with tryptophan at $\mathrm{C} 139 \mathrm{~T}$ ) substitution was found in the PAX9 paired domain at the N-terminal region and assumed to affect the specificity of targeted DNA [4]. A two-residue mutation (R28P) located in the N-terminal region of PAX9 reduces DNA binding ability [4]. The last missense mutation, G51S (glycine to serine at amino acid 51) is caused by substitution of guanine to adenine at codon 151 of the PAX9 paired domain and is found between the $\mathrm{C}$ and terminal region [4].

The phenomenon whereby a diploid organism has only a single functional copy of a gene while the other copy is inactivated by mutation and the single, active copy of the gene does not produce enough of the gene product, in this case the protein, to bring about a wild-type condition, leading to a diseased or an abnormal state is called haploinsufficiency. In two studies, haploinsufficiency in the PAX9 gene caused by different mechanisms was investigated: one study found a deletion of the entire PAX9 gene, whereas another found a nucleotide substitution in the first position of the ATG start codon, which has been hypothesized to abolish protein expression [4]. However, Kapadia suggested that the absence of gene function could determine haploinsufficiency of PAX9 [17]. An example of such a mechanism is a missense mutation in the PAX9 gene at amino acids S43K and G6R detected in two Chinese patients with non-syndromic tooth hypodontia [3]. Family members and patients affected by oligodontia and other dental anomalies were carrying a nonsense transition mutation at $\mathrm{C} 175 \mathrm{~T}$, resulting in replacement of arginine 59 with a stop codon and leading to haploinsufficiency in the PAX9 gene due to premature chain termination [3].

Most mutations in the PAX9 gene alter the DNA binding ability of the PAX9 paired domain and decrease its transcriptional activity [4]. Most of the deletion, frameshift, and missense termination mutations are related to CMT in both the primary and permanent dentition, whereas missense mutations cause CMT in the permanent dentition only and show a milder phenotype than nonsense and frameshift mutations $[4,15]$.

\section{Epigenetics in Congenital Missing Teeth}

The term epigenetics is broadly defined as processes by which the genotype gives rise to the phenotype through a combination of environmental and genetic factors; the interactive processes that occur between the local tissue level and cells during dental development are termed epigenetic events, in addition to those that may operate directly on DNA [18]. The way a cell reads the genetic code can be changed through epigenetics: the DNA message may be altered in a number of ways, including by adding notes to or tags to the DNA structures or bases that DNA wraps around to change the activity within a gene [19]. These tags sometimes can either activate or repress gene expression and the resulting protein production [19]. Histone modification and DNA methylation are two of the most common epigenetic alterations; they affect chromatin inactivation, specific gene expression related to 
embryonic growth, cell differentiation, and cancer progression through the timing of interactive signaling and the spatial arrangement of cells [20]. For example, it has been found that dental stem cell differentiation is regulated by histone demthylase [20]. The role of epigenetic factors during odontogenesis as local epigenetic influences include cell-matrix and cell-cell interactions and their effects on differential cell proliferation during dental development and asymmetrical growth [18]. This theory explains monozygotic (MZ) twin pairs who were confirmed in one study to have the same genotypes with distinct differences in dental features, including the location and/or number of CMT [18].

In another study, the genetic mutations in a family with CMT had not yet been seen, yet the presence of epigenetic changes related to activity of the PAX9 and MSX1 genes could be found.5 Morphological studies in mice indicate various defects in craniofacial development, including dental agenesis, which may result from absence of the PAX9 or MSX1 genes [5]. Moreover, epigenetic regulation should appear at predetermined times of maxillofacial development in reversible form, causing, for example, the isolated form of dental agenesis or the syndromic type [5]. There is still little literature about epigenetic factors in CMT. Methylation of the MSX1 and PAX9 genes has been associated with cancer development; however, this was not related to dental agenesis [5]. Another study showed evidence that a low level of PAX9 expression may affect tooth morphogenesis and manifests in mice as the isolated form of oligodontia [5]. These lines of evidence prove that epigenetic factors controlling gene expression can cause CMT [5].

Dentoalveolar defects may be generated from changes in post-transcriptional activity of PAX9 and MSX1 [5]. MSX1 antisense RNA can regulate the activity of RNA produced from MSX1 [5]. The major role of the molecule is to affect the regulation of craniofacial development, especially that of alveolar bone formation [5]. The interaction between the PAX9 and MSX1 genes may also originate from other elements [5]. The relationship between these two genes during odontogenesis engages another potential level of regulation [5]. Therefore, direct interference at the DNA or post-transcriptional level due to epigenetic factors can alter phases of tooth development based on these interactions [5].

The literature reveals that alteration in methylation status affects a network of interconnected signaling pathways, which include neurogenesis and tooth development, and these pathways may be altered by factors other than changes in the methylation of DNA or acetylation of histones. Moreover, the exact mechanism by which methylation influences signal pathways is still unclear [20]. Further investigation is needed on the influence of epigenetic factors on the PAX9 and MSX1 genes that could manifest as the isolated type of dental agenesis in humans to explain the entire mechanism of CMT [5].

\section{Conclusion}

Dental agenesis or CMT is a common multifactorial dental anomaly usually found in the permanent dentition with a higher prevalence in women [1]. This anomaly manifests in a genetically and phenotypically heterogeneous manner. One of the major etiologies is a genetic trait. Over 300 genes are known to be involved in the development of normal dentition and have roles in a several different manifestations 
of hypodontia [21]. This paper discussed only a few defects that are commonly found in the autosomal dominant non-syndromic type of agenesis related to the MSX1 and PAX genes. However, the majority of hypodontia patients who possess mutations in the MSX1 or PAX9 genes may also have other gene mutations. The molecular genetics of related genes, such as MSX1, PAX9, AXIN2, EDA, and others, may also have a major role in minimizing the risk of inherited genetic tooth anomalies [7]. There is a possibility that other specific hypodontia genes exist and will be identified in the future with the contribution of molecular genetic research [6]. By reducing gene dosage, altering gene interactions, and other post-transcriptional modes of modulation, epigenetic factors can be involved in and explain the mechanism of congenital missing teeth associated with systemic anomalies or isolated CMT [5]. Further investigation of genes and epigenetic screening should be carried out for better diagnosis and prevention and promising treatment for patients with congenital missing teeth [7]. Pediatric dentists are encouraged to contribute to future research and overcome the problems inherent in CMT by understanding the basic concepts of its causation.

\section{References}

1 Rakhshan V. Congenitally missing teeth (hypodontia): A review of the literature concerning the etiology, prevalence, risk factors, patterns and treatment. Dent Res J. 2015;12(1):1-13.

2 Arte S, Pirinen S. Hypodontia. Orphanet Encyclopedia. May 2004: 1-7.

3 Abu-Hussein M, Watted N, Yehia M, Proff P, Iraqi F. Clinical genetic basis of tooth agenesis. J Dent Med Sci. 2015. 2015;14(12):68-77.

4 Pemberton TJ, Das P, Patel PI. Hypodontia: genetics and future perspectives. Brazilian J Oral Sci. 2005;4(13):695-706.

5 Tallón-Walton V, Manzanares-Céspedes MC, Carvalho-Lobato P, Valdivia-Gandur I, Arte S, Nieminen P. Exclusion of PAX9 and MSX1 mutation in six families affected by tooth agenesis. A genetic study and literature review. Med Oral Patol Oral Cir Bucal. 2014;19(3): e248.

6 Shimizu T, Maeda T. Prevalence and genetic basis of tooth agenesis. Jpn Dent Sci Rev. 2009;45(1):52-8.

7 Shahid M. Genetic Anomalies and Tooth Agenesis: Review Article. J Gene Rev Biomed Pharmacol J. 2013;6(1):9-16.

8 Vastardis H. The genetics of human tooth agenesis: new discoveries for understanding dental anomalies. Am J Orthod Dentofacial Orthop. 2000;117(6):650-6.

9 Yin W, Bian Z. The gene network underlying hypodontia. J Dent Res. 2015;94(7):87885.

10 Chhabra N, Goswami M, Chhabra A. Genetic basis of dental agenesis-molecular genetics patterning clinical dentistry. Med Oral Patol Oral Cir Bucal. 2014;19(2): e112.

11 Vasudev J. A study of non-syndromic hypodontia in a high-risk population (Doctoral dissertation, University of Birmingham).

12 Center LHN. Mutations and Health. J. Mutat. Health. Genetics Home Reference. https://ghr.nlm.nih.gov/. Published 2017.

13 Briscoe PR. Genetic components determining familial hypodontia. University of Missouri-Kansas City; 2008.

14 New South Wales HG. Gene Mutations. In: Centre for Genetics Education. J. Gene. Mutat. Gene. Educ. Health Government of New South Wales; 2016:1-3. www,genetics.edu.au. 
15 Bailleul-Forestier I, Molla M, Verloes A, Berdal A. The genetic basis of inherited anomalies of the teeth: Part 1: Clinical and molecular aspects of non-syndromic dental disorders. Eur J Med Genet. 2008;51(4):273-91.

16 Dudlicek LL, Gettig EA, Etzel KR, Hart TC. Status of genetics education in US dental schools. J Dent Educ. 2004;68(8):809-18.

17 Militi D, Militi A, Cutrupi MC, Portelli M, Rigoli L, Matarese G, Salpietro DC. Genetic basis of non-syndromic hypodontia: a DNA investigation performed on three couples of monozygotic twins about PAX9 mutation. Eur J Paediatr Dent: official journal of European Academy of Paediatric Dentistry. 2011;12(1):21-4.

18 Townsend G, Harris EF, Lesot H, Clauss F, Brook A. Morphogenetic fields within the human dentition: a new, clinically relevant synthesis of an old concept. Arch Oral Biol. 2009;54: S34-44.

19 New South Wales HG. Epigenetics. In: Centre for Genetics Education. J Epigenetics. Gene. Educ. Health Government of New South Wales; 2015:1-7. www,genetics.edu.au.

20 Wang J, Sun K, Shen Y, Xu Y, Xie J, Huang R, Zhang Y, Xu C, Zhang X, Wang R, Lin Y. DNA methylation is critical for tooth agenesis: implications for sporadic non-syndromic anodontia and hypodontia. Sci Rep. 2016; 6:19162.

21 Galluccio G, Castellano M, La Monaca C. Genetic basis of non-syndromic anomalies of human tooth number. Arch Oral Biol. 2012;57(7):918-30. 